\title{
Reproducción precaria, soporte de la sustentabilidad capitalista
}

\section{Guadalupe Margarita González Hernández José Roberto González Hernández}

Reproducción precaria es un constructo teórico-metodológico de un fenómeno estructural múltiple que valora dimensiones, formas e intensidades de exclusión en el proceso de desarrollo y disfrute de sus beneficios. Por medio del Índice de Reproducción Precaria (IRP) se identifican distintos grados de insatisfacción de necesidades biológicas y sociales de los individuos y sus familias que aluden a su organización privada como resultado directo o indirecto de las relaciones sociales de producción capitalista. 


\section{REPRODUCCIÓN}

SOCIAL Y PRECARIEDAD

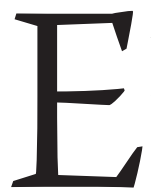

1 proceso de reproducción social precario está relacionado, principalmente, a factores económicos y sociales, en alusión más a cuestiones estructurales de organización que a contextos temporales. El cambio de la estructura productiva hacia la flexibilización laboral, con la consecuente precarización, y el desentendimiento del Estado sobre la responsabilidad social sustentan un proceso de reproducción social precario y el desarrollo de estrategias familiares de sobrevivencia: migración, constitución de formas familiares no nucleares (familias extensas, unifamiliares y jefatura femenina), formas no capitalistas de obtención de ingresos, entre otras. ${ }^{1}$

Aunque la reproducción social precaria puede verse desde distintas visiones, interesa la reproducción de la fuerza de trabajo y su familia, pues permite analizar el desgaste físico y psicosocial del trabajador, que cubre la reposición generacional...

y debe ser remitido a la reconstrucción del conjunto de capacidades en el nivel societal. Las características que asumen los procesos de trabajo y de producción son relevantes para entender la manera en que se reproduce la fuerza de trabajo con características específicas en términos de calificación, docilidad, adaptabilidad, rotatividad y eventualidad. Pero también los rangos que asumen la organización familiar y los mecanismos de socialización, aprendizaje y control que se dan en las unidades domésticas, las organizaciones sindicales y la escuela cumplen un papel central en este proceso. ${ }^{2}$

El proceso de restructuración de la economía promovido para mantener la tasa de ganancia, basado en la introducción de nuevas tecnologías, provocó la flexibilización en el proceso productivo y afectó la contratación de fuerza de trabajo en dos formas. La primera, mediante la intensificación del proceso laboral, salarios más bajos, jornadas más largas y ausencia de prestaciones sociales. Con el paso del tiempo, los trabajadores que permanecieron ocupados se vieron imposibilitados para ahorrar y cubrir actividades de ocio; inclusive, el salario ya no cubre las necesidades básicas. El tiempo de descanso se redujo con el aumento de las jornadas laborales al tomar horas extras para acceder a un ingreso adicional y al cancelar o reduccir el tiempo dedicado anualmente a la reposición de fuerzas. La segunda, mediante el despido masivo de trabajadores industriales que ya no fueron requeridos y se vieron inmersos en el desempleo, subempleo y autoempleo en sectores menos rentables y con menor productividad, como los servicios que dieron cabida a una inmensa precarización del trabajo con jornadas interminables, ingresos magros y nulas posibilidades de prestaciones sociales que permitan su reposición ${ }^{3}$ y reproducción como seres humanos y clase social.

Derivado de la diferente organización del capital y la dinámica de las economías subdesarrolladas, el intercambio mercantil propicia efectos expansivos en la producción y el empleo de los países desarrollados. En cambio, en las naciones subdesarrolladas acontece una transferencia neta de valor a naciones desarrolladas que se traduce en límites a su propia acumulación y afecta su capacidad de autoexpansión e invariablemente restringe sus posibilidades de absorber fuerza de trabajo. La sobrepoblación, inherente a los países subdesarrollados, se registra de manera ampliada al constituirse por a) la sobrepoblación relativa o ejército industrial de reserva, considerado como exceso de población obrera respecto a las exigencias medias del capital para realizar su proceso de valorización, ${ }^{4}$ y b) la sobrepoblación absoluta formada por una masa de población trabajadora en cuanto a las necesidades de acumulación. ${ }^{5}$

Si se observa la pesada carga poblacional que sobrellevan las sociedades subdesarrolladas, se comprenderá la proliferación de las formas de organización del trabajo no capitalistas como válvulas de escape a sus contradicciones. $^{6}$

La reproducción precaria se considera un fenómeno estructural en relación al desarrollo socioeconómico fundamentado en la lógica de la relación capital-trabajo. Bajo este fenómeno de reproducción precaria se encuentra toda la población que presenta los siguientes rasgos: $i$ ) ingresos derivados de actividades laborales; ii) alimentación como rubro dominante en el gasto; iii) ingreso insuficientes, restricción en el gasto y uso regular del crédito; iv) ausencia de ahorro en líquido y propensión a «invertir» en 
vivienda; $v$ ) escasez de activos para hacer frente a variaciones drásticas en el patrón de ingresos y gastos; vi) vulnerabilidad habitacional y hacinamiento; vii) prioridad en el equipo doméstico con fines de esparcimiento; viii) relaciones familiares y sociales conflictivas, y $i x$ ) pasividad en el ejercicio de sus derechos fundamentales.?

\section{REPRODUCCIÓN PRECARIA} URBANA Y SU MEDICIÓN
Bajo la dinámica capitalista, la organización socioeconómica y política de las ciudades integra a la población precaria de manera desigual en la producción y distribución de bienes y servicios; al tiempo en que la excluye, total o parcialmente, del consumo y disfrute de bienes y servicios, también lo hace de la participación en asuntos públicos.

El Índice de Reproducción Precaria (IRP) ${ }^{8}$ pretende medir, a través de grados de intensidad, el impacto global de las carencias de los pobladores urbanos más sensibles a las desigualdades sociales y económicas del entorno. La reproducción precaria está constituida por cuatro dimensiones: condiciones de vivienda, ingresos monetarios, educación y segregación socioespacial.

Las personas y familias (o miembros que integran un hogar) que carecen de condiciones materiales mínimas en la vivienda y el entorno inmediato o que padecen restricciones para acceder a recursos o servicios públicos urbanos para satisfacer sus necesidades biológicas y sociales sufren reproducción precaria. En ello inciden los ingresos monetarios que resultan insuficientes para acceder al consumo de bienes y servicios indispensables para vivir en condiciones sociales dignas. Para fines analíticos, sólo interesan los ingresos provenientes de actividades laborales remuneradas, pues las transferencias, subsidios o remesas aminoran el deterioro de las condiciones materiales de las familias y diluyen la incapacidad de la estructura productiva para dotar de empleo a la población y sus familias con menores capacidades y recursos.

La población y las familias privadas del servicio educativo se integran al cada vez más dinámico y menos regulado mercado de trabajo en condiciones de vulnerabilidad, a expensas de ingresos magros. Esto repercute en un impedimento para el disfrute de los bienes culturales y un mejor ejercicio de los derechos civiles y políticos, lo cual da pie a una mayor exclusión social. ${ }^{9}$

Los pobladores urbanos con menores recursos monetarios y educativos y con hábitos de consumo deteriorados están obligados, estructuralmente, a situar su residencia en lugares con mayor aislamiento social y tienen menores posibilidades de acceder y disfrutar los recursos que ofrece la ciudad. La segregación socioespacial urbana es resultado de las decisiones de localización (acceso a una vivienda, a un habitus ${ }^{10}$ de consumo ${ }^{11}$ ) y los pobladores en condiciones precarias reproducen las prácticas del grupo al que pertenecen. ${ }^{12}$
De la aplicación en varios años del IRP $^{13}$ en la ciudad Zacatecas-Guadalupe (CZG), se puede rescatar que a pesar de que no existieron condiciones alarmantes de precariedad en el periodo analizado, sí hay conglomerados en los que se concentraron personas que no sólo experimentaron una marcada situación carencial, sino que la han acentuado con el paso del tiempo. La complejidad se hace presente: se cuenta con energía eléctrica, pero se carece de espacios en la vivienda para mantener un estado emocional estable; se cuenta con educación básica, pero no se tiene empleo o se reciben ingresos insuficientes. «La dotación de servicios públicos ya no explica el grado de aislamiento social y económico» que vive la población zacatecana, «sino la ausencia de capacidades tanto económicas como sociales para poder disfrutar de los bienes y servicios que ofrece» ${ }^{14}$ la ciudad. Motivo suficiente para reformular el índice.

\section{Hacia el Replanteamiento del ÍNDICE DE REPRODUCCIÓN PRECARIA}

Las deficiencias del IRP aluden, principalmente, a la intensidad de ciertos fenómenos asociados a condicionamientos estructurales de la sociedad capitalista que las bases de datos difícilmente pueden cuantificar. No obstante, la restructuración productiva involucra a las personas y sus familias a través de distintos flancos, sobre todo por la profundización del desempleo. Ya el IRP aludía a la abundancia de sobrepoblación, que lejos de ser un ejército industrial de reserva (que supone un posible empleo en tiempo y espacio futuro), es un fenómeno estructural que da cuenta de población excluida en el presente y en el futuro del mercado laboral dado que la maximización de la ganancia se basa, entre otros mecanismo, en ahorro del factor trabajo y su remuneración. ${ }^{15}$

El despido masivo de trabajadores en el sector industrial, para ser sustituidos por otros más capacitados o por maquinaria, in- 
cita a los desocupados a buscar opciones en sectores menos remunerados y menos productivos. El sector terciario se vuelve idóneo para acapararlos, ${ }^{16}$ pero a costa de la reducción o nula existencia de prestaciones ligadas a la relación capital-trabajo. En países como México, la seguridad social, a pesar de ser un derecho social, no está sustentada en un precepto legal. Por lo contrario, se liga a la contratación e inserción al mercado laboral. $\mathrm{Al}$ reducir el número de personas asalariadas en sectores como el industrial, la cobertura de seguridad social se reduce. ${ }^{17}$

Aunque la seguridad social, especialmente el acceso a servicios de salud, se convierte en un elemento fundamental para mejorar los estándares de vida de los pobladores mexicanos, la restructuración productiva ha incitado el fenómeno contrario a causa de la reducción de contratación de trabajadores basados en la relación capital-trabajo y la reducción de percepciones económicas ligadas al salario. El trabajo a destajo, el autoempleo o subempleo no permiten ser registrados en el sistema de seguridad social actual. Por tanto, las familias con elevados indicadores de reproducción precaria empeoran más su condición al no poseer seguridad social, en particular servicios de salud pública.

La pasividad en el ejercicio de los derechos fundamentales se planteó desde el IRP clásico, no obstante sólo se midió el aislamiento geográfico y funcional y el acceso reducido o nulo a los recursos que ofrece la ciudad. ${ }^{18}$ No es suficiente. La Encuesta Nacional sobre Cultura Política y Prácticas Ciudadanas (ENCUP) de la Secretaría de Gobernación $(\text { Segob })^{19}$ indicó que la mayor parte de los mexicanos se informa a través de la televisión (60\% en 2005, para llegar a 87\% de los encuestados en 2008). Este dato tiene implicaciones importantes en la forma y tipo de participación. El control monopólico de los medios masivos de comunicación, como la televisión, impulsa cierto tipo de cultura política que induce a un comportamiento cívico pasivo reducido al ejercicio del sufragio. ${ }^{20}$ Las familias y personas que no se informan por diversos medios - salvo por la televisión-, sobre lo que pasa en su comunidad, consolidan su pasividad y reducen la posibilidad de superar la reproducción precaria en la que viven.

Finalmente, el desempleo abierto, en la intensificación de la restructuración productiva actual, sigue siendo un factor característico, a pesar de estar oculto por estrategias como el subempleo o autoempleo. El desempleo afecta a las familias, al igual que las otras estrategias de sobrevivencia (transferencias, subsidios, remesas), al reducir los ingresos percibidos para la satisfacción de las necesidades y se convierte en un elemento de conflicto entre sus miembros por el dominio y poder de la organización familiar.

Debido a las deficiencias mostradas por el IRP clásico o, más concretamente, a la progresiva complejidad de la reproducción precaria, se propone un Índice de Reproducción Precaria reformulado (IRPr) que introduce nuevos elementos de análisis, además de los involucrados en el IRP. Dichos elementos se refieren a $i$ ) la ausencia de derechohabiencia a seguridad social para la familia; ii) la capacidad de los miembros de la familia a informarse a través de un medio de comunicación, y iii) el desempleo abierto de algún miembro de la familia en edad de trabajar. Estos elementos se enmarcan en las dimensiones de empleo, salud y segregación socioespacial. El mismo procedimiento se aplicó para el periodo 2005-2010 en la CZG. ${ }^{21}$

Al comenzar la primera década del siglo xxi, la reproducción precaria, a pesar de disminuir su incidencia en la CZG, siguió siendo explicada por la dinámica de la sociedad capitalista basada en la disminución del trabajo sustentado en la estabilidad laboral. La precarización del trabajo apoyado en la baja productividad, bajos ingresos y nulas prestaciones sociales influyó fuertemente en la dinámica de la estructura familiar de las personas y su organización física y social. Dada la ausencia de un mercado de trabajo estable con seguridad en el empleo y de una institución pública que defienda sus intereses (el Estado se dedica a actividades que regulan la eficiencia del sistema económico), los pobladores de la CZG asumieron, en el ámbito familiar, los costos de la restructuración productiva. Es en la microesfera privada familiar donde la dominación del capital sobre el trabajo hace estragos al limitar el acceso a los recursos educativos, culturales, económicos y políticos que ofrece la ciudad.

Según los resultados del IRPr en la CZG, el ingreso restringido por realizar trabajos precarios y, ahora, el desempleo, explican principalmente la reproducción precaria de los miembros del hogar. La educación, aunque con menor importancia, sigue manteniendo su influencia, mientras que factores más relacionados a la obtención de servicios públicos (dotación de agua potable, drenaje y energía eléctrica) son cada vez menos relevantes. Por ello, aunque la magnitud del fenómeno ha decrecido, se profundizó y complejizó, sin posibilidad de solucionarse si se mantiene el modelo de desarrollo actual. La situación precaria se reprodujo al ritmo de la intensificación de las relaciones capitalistas en los distintos ámbitos de la esfera social. 
1 Gioconda Herrera, «Precarización del trabajo, crisis de reproducción social y migración femenina: ecuatorianas en España y Estados Unidos» en Herrera Gioconda (editor), La persistencia de la desigualdad: género, trabajo y pobreza en América Latina, Quito, FLACso, CONAMU, Secretaría Técnica del Frente Social, 2006, pp. 199-223.

2 Orlandina de Oliveira y Vania Salles, «Reflexiones teóricas para el estudio de la reproducción de la fuerza de trabajo», Argumentos, número 4, 1988, pp. 19-43.

3 Arturo Huerta, Economía mexicana más allá del milagro, México, Diana, 1991; Arturo Huerta, La continuidad del bajo crecimiento de la economía mexicana, Málaga, Eumed, 2007.

4 Karl Marx, El Capital, México, Siglo xxi, 1985.

5 Mario Margulis, «Reproducción social de la vida y reproducción del capital», Nueva Antropología, año IV, números 13-14, 1980, pp. 47-64; Rodolfo García (coordinador), Desarrollo humano sustentable, derechos humanos y seguridad humana en el municipio de Zacatecas, Zacatecas, Universidad Autónoma de Zacatecas, 2012.

6 Guadalupe González, Irma Acosta, José González, José Ramírez y Víctor Figueroa, Reproducción precaria familiar. Conceptualización y evidencias en Zacatecas-Guadalupe (1990-2004), Zacatecas, Fondo Mixto CONACYT-GODEZAC, Universidad Autónoma de Zacatecas, 2007; Mario Margulis, op. cit.

7 Guadalupe González et al., op. cit., p. 147.

8 Guadalupe González, «Reproducción precaria de las familias en la zona conurbada Zacatecas-Guadalupe, México (19902004)» en Carlos Lasarte (director), Perspectivas del Derecho de Familia en el siglo XXI, Sevilla, Instituto de Desarrollo y Análisis del Derecho de Familia en España, 2004, pp. 1-25; Guadalupe González, «Propuesta metodológica para medir la reproducción precaria», Boletín del Sistema Nacional de Información Estadística y Geográfica, volumen 2, número 2, mayo-agosto, 2009, pp. 101-126; Guadalupe González e Irma Acosta, «Reproducción precaria familiar de las ciudades mexicanas: el caso de Zacatecas» en Nau Niño et al. (coordinador), Antología de Estudios Regionales. Fomento de los estudios territoriales en Iberoamérica, Acapulco, GEотесH, Universidad Autónoma de Guerrero, 2009, pp. 1-25; Guadalupe González y José González, «Beyond Poverty: Precarious Reproduction of Mexican Families: A Case Study in the Urban Area of Zacatecas-Guadalupe 1990-2004» en Clemens Sedmak y Thomas Böhler (editores), Perspectives in Poverty Alleviation, Vienna-Münich, LiT VerLag GMBH y Co., 2007, pp. 137-156; Guadalupe González et al., Reproducción precaria familiar. Conceptualización y evidencias en Zacatecas-Guadalupe (1990-2004), op. cit.

9 Guadalupe González, «¿Comportamientos coyunturales o castigos sociales? Esbozos sobre elecciones municipales en Zacatecas-Guadalupe» en Rubén Ibarra (coordinador), Zacatecas y su contexto actual, México, Taberna Editores, 2010, pp. 15-39.

10 Reproducción de prácticas propias al segmento social de conocimiento al que se siente pertenecer. Tito Alegría, «Segregación socioespacial urbana. El ejemplo de Tijuana», Estudios Demo- gráficos y Urbanos, volumen IX, número 2, mayo-agosto, 1994, p. 419.

11 Pierre Bourdieu, El sentido práctico, Madrid, Taurus, 1991; Pierre Bourdieu, La distinción. Criterio y bases sociales del gusto, México, Taurus, 2003; Pierre Bourdieu, Capital Cultural, escuela y espacio social, México, Siglo xxi, 2003; Tito Alegría, op. cit.

12 Pierre Bourdieu El sentido práctico; Pierre Bourdieu, La distinción. Criterio y bases sociales del gusto; Pierre Bourdieu, Capital Cultural, escuela y espacio social.

13 En los años de 1990, 1995, 2000, 2005 y 2010. Los resultados pueden verse en Guadalupe González «Reproducción precaria de las familias en la zona conurbada Zacatecas-Guadalupe, México (1990-2004)»; Guadalupe González, «Propuesta metodológica para medir la reproducción precaria»; Guadalupe González e Irma Acosta, «Reproducción precaria familiar de las ciudades mexicanas: el caso de Zacatecas»; Guadalupe González y José González «Beyond Poverty: Precarious Reproduction of Mexican Families: A Case Study in the Urban Area of Zacatecas-Guadalupe 1990-2004»; Guadalupe González et al., Reproducción precaria familiar. Conceptualización y evidencias en Zacatecas-Guadalupe (1990-2004); Guadalupe González y José González, «Complejidad de la reproducción precaria. Replanteamiento metodológico en ZacatecasGuadalupe, México 1990-2010», Memoria del XII Congreso de la Asociación Latinoamericana de Escuelas de Urbanismo y Planeación, Toluca, 2012, pp. 1-25.

14 Guadalupe González et al., Reproducción precaria familiar. Conceptualización y evidencias en Zacatecas-Guadalupe (1990-2004), p. 139.

15 Idem.

16 Jürgen Weller, «Tendencias del empleo en los años noventa en América Latina y el Caribe», Revista de la CEPAL, número 72, diciembre, 2000; Jürgen Weller, «El empleo terciario en América Latina: entre la modernidad y la sobrevivencia», Revista de la CEPAL, número 84, diciembre, 2004.

17 Antonio Guzmán, Seguridad Social y sistema de pensiones: énfasis en el caso de México, México, Universidad Autónoma de Zacatecas, 2012.

18 Guadalupe González et al., Reproducción precaria familiar. Conceptualización y evidencias en Zacatecas-Guadalupe (19902004).

19 Segob, Tercera Encuesta Nacional sobre Cultura Política y Prácticas Ciudadanas (ENCUP), México, 2005; SEgoB, Cuarta Encuesta Nacional sobre Cultura Política y Prácticas Ciudadanas (ENCUP), México, 2008.

20 Rubén Ibarra, La crisis democrática. Marginación y abstencionismo electoral en Zacatecas 1998-2007, Zacatecas, Universidad Autónoma de Zacatecas, 2009; Rodolfo García (coordinador), op. cit.

21 Los resultados pueden verse en Guadalupe González y José González, «Complejidad de la reproducción precaria. Replanteamiento metodológico en Zacatecas-Guadalupe, México 1990-2010». 